\title{
Kluyveromyces marxianus
}

National Cancer Institute

\section{Source}

National Cancer Institute. Kluyveromyces marxianus. NCI Thesaurus. Code C114256.

A dimorphic, homothallic and hemiascomycetous yeast in the phylum Ascomycota. This species is respiro-fermentative, Crabtree negative, thermotolerant, and assimilate lactose and insulin. This species also has a characteristically high growth rate and secretory capacity. K. marxianus is a biocatalyst and used industrially to produce betagalactosidase, beta-glucosidase, inulinase, and polyg alacturonases. It is also used as baker's yeast and an anticholesterolemic agent. 